DOI: $10.5433 / 1981-8920.2017 v 22 n 1 p 263$

\title{
O COMPARTILHAMENTO DO CONHECIMENTO E A INOVAÇÃO NOS RELACIONAMENTOS INTERORGANIZACIONAIS DO TIPO TERCEIRIZAÇÃO DE TECNOLOGIA DA INFORMAÇÃO
}

\author{
INTERCAMBIO DE CONOCIMIENTOS E INNOVACIÓN \\ EN LAS RELACIONES INTERORGANIZATIONAL TIPO \\ DE OUTSOURCING TECNOLOGÍA DE LA \\ INFORMACIÓN
}

\author{
Roberta Rodrigues Faoro * \\ Mírian Oliveira** \\ Marcelo Faoro de Abreu***
}

\begin{abstract}
RESUMO:
Introdução: este artigo apresenta uma análise do compartilhamento do conhecimento e da inovação nos relacionamentos interorganizacionais do tipo terceirização de Tecnologia da Informação. Objetivo: analisar a existência de relação entre o compartilhamento do conhecimento e a inovação nas relações interorganizacionais do tipo terceirização de tecnologia da informação (TI). Metodologia: pesquisa é exploratória, de natureza qualitativa e utilizando como estratégia o estudo de casos múltiplos, onde foram analisadas 12 empresas em ambientes de terceirização de TI, sendo 4 empresas fornecedoras de TI e 8 empresas clientes de TI. Os dados foram coletados através de entrevistas semiestruturadas, documentos e observação direta. Resultados: pode-se destacar que o compartilhamento do conhecimento mostrou contribuir para a existência de inovação no ambiente de terceirização de $\mathrm{Tl}$. As empresas fornecedoras priorizam a codificação do conhecimento compartilhado, enquanto que as clientes preferem o compartilhamento de conhecimento através de

*Doutora em Administração pela Associação da Pontifícia Universidade Católica do Rio Grande do Sul, PUCRS e da Universidade de Caxias do Sul, UCS. Professora da Universidade de Caxias do Sul - UCS. E-mail: roberta.faoro@ucs.br

**Doutora em Administração pela Universidade Federal do Rio Grande do Sul, UFRGS. Professora da Pontifícia Universidade Católica do Rio Grande do Sul, PUCRS. E-mail: marcelo.faoro@ucs.br

***Doutor em Administração pela Universidade Federal do Rio Grande do Sul, UFRGS. Professor da Universidade de Caxias do Sul - UCS. E-mail: marcelo.faoro@ucs.br
\end{abstract}


Roberta Rodrigues Faoro, Mírian Oliveira, Marcelo Faoro de Abreu O compartilhamento do conhecimento e a inovação nos relacionamentos interorganizacionais do tipo terceirização de tecnologia da informação

conversas informais. Identificou-se que os motivos para a terceirização de TI podem estar relacionados com o compartilhamento do conhecimento e a inovação. Conclusões: esta pesquisa contribui para o avanço do conhecimento acerca dos fenômenos compartilhamento de conhecimento e inovação em ambientes de terceirização da TI.

Palavras-chave: Compartilhamento do conhecimento interorganizacional. Inovação. Tecnologias da informação e comunicação.

\section{INTRODUÇÃO}

A terceirização de Tecnologia da Informação ( $\mathrm{TI})$, esta tendo grande notoriedade nos últimos anos, em virtude dos crescentes investimentos das empresas nesta área, pois, segundo a pesquisa da empresa de consultoria Gartner Group, os gastos globais para a terceirização de TI foram projetados para alcançar US\$ 287 bilhões em 2013 (HAN; MITHAS, 2014). Ainda, cabe destacar que, o crescimento do mercado relacionado à terceirização de TI terá um crescimento anual de 5,4\% no mercado global até 2017 (GARTNER, 2013). A esse respeito, o Brasil está seguindo a tendência mundial, segundo o estudo de Kearney (2014), o País subiu quatros posições dos principais mercados para outsourcing em tecnologia. O Brasil, que em 2013 ocupava o $12^{\circ}$ lugar, pulou em 2014 para o $8^{\circ}$ posto, ficando entre os dez maiores países para outsourcing dessa atividade, atrás da Índia, China, Malásia, México, Indonésia, Tailândia e Filipinas.

"A terceirização de $\mathrm{TI}$ tem apresentado um crescimento contínuo desde a sua adoção de destaque pela Kodak há 20 anos” (QI; CHAU, 2012, p. 859). Associado a isso, a literatura vem destacando a importância da relação entre o fornecedor e o cliente como um indicador para os resultados bem-sucedidos (VIVEK; SUSHILL, 2014). Neste sentido, vários processos e/ou atributos do relacionamento de terceirização como compatibilidade cultural, confiança, comunicação, podem ser descritos em um contrato, mas em última análise, a relação deve ser desenvolvida e alimentada pelos indivíduos que executam as atividades de terceirização. Logo, o desafio reside principalmente na forma de 
Roberta Rodrigues Faoro, Mírian Oliveira, Marcelo Faoro de Abreu O compartilhamento do conhecimento e a inovação nos relacionamentos interorganizacionais do tipo terceirização de tecnologia da informação

compartilhamento do conhecimento entre $\mathrm{o}$ fornecedor $\mathrm{e} O \mathrm{c}$ cliente. $\mathrm{A}$ terceirização implica que o compartilhamento do conhecimento seja interorganizacional, considerando o objeto terceirizado. Consequentemente, os canais para o compartilhamento de conhecimento serão mais formais e impessoais na terceirização (GOTTSCHALK, 2006).

O compartilhamento de conhecimento é relacionado com a geração de inovações (LEE; LEE; KANG, 2005). Associado a isto, identifica-se que o conhecimento externo pode ser um recurso relevante para a inovação nas empresas (WANG; HAN, 2011). O compartilhamento do conhecimento é um dos principais fatores para o sucesso da terceirização, já a capacidade da organização de aprender ou adquirir os conhecimentos necessários de outras organizações é uma fonte fundamental para o sucesso do compartilhamento do conhecimento (LEE, 2001). Consonante a isto, Lacity et al. (2010) concluem, em seu estudo, que o compartilhamento do conhecimento eficaz sempre foi positivamente associado com melhores resultados na terceirização de TI. No entanto, a forma como o compartilhamento do conhecimento interorganizacional contribui para a inovação no contexto de terceirização de software ERP é pouco conhecida. Diante disso, o objetivo deste estudo é analisar a relação entre o compartilhamento do conhecimento e a inovação nas relações interorganizacionais do tipo terceirização de software ERP.

\section{REFERENCIAL TEÓRICO}

\subsection{Ambiente de Estudo: Terceirização de TI}

As decisões de terceirização têm sido frequentemente consideradas sob o ponto de vista da teoria dos custos de transação - TCT (LEE; MIRANDA; KIM, 2004). A TCT está baseada na preocupação com os custos de transação, ou seja, os custos associados à garantia de uma boa execução da transação. No entanto, recentemente, a terceirização de TI tem sido reconhecida como uma estratégia para aumentar a eficiência $e$ reduzir os custos das implementações dos sistemas de informação (YOON; IM, 2005). A estratégia 
Roberta Rodrigues Faoro, Mírian Oliveira, Marcelo Faoro de Abreu O compartilhamento do conhecimento e a inovação nos relacionamentos interorganizacionais do tipo terceirização de tecnologia da informação

de terceirização devidamente implementada reúne conhecimento da indústria de $\mathrm{TI}$, cria sistemas que ajudam as organizações a adquirir e manter uma vantagem competitiva e oferece um melhor serviço a um custo menor (SENGUPTA; ZVIRAN, 1997). Em ambientes de terceirização de TI, os requisitos dos clientes e feedbacks são essenciais para o desenvolvimento de aplicações dos sistemas de informação e a melhoria da qualidade de serviços dos fornecedores ou empresas de serviços de TI (YOON; IM, 2005).

Um dos principais focos de discussão sobre a terceirização nas organizações é a redução de custos. Enquanto a redução de custos é, definitivamente, um dos objetivos, as organizações estão cada vez mais terceirizando suas operações, a fim de beneficiar-se das habilidades complementares que estão disponíveis na empresa externa (BANDYOPADHYAY; PATHAK, 2007). Assim sendo, o compartilhamento do conhecimento tem uma estreita relação com a terceirização bem-sucedida (MANI; BARUA; WHINSTON, 2010). A qualidade do relacionamento com o fornecedor é um influenciador chave para permitir o compartilhamento de conhecimento que contribui para a obtenção de benefícios de terceirização (POPPO; ZHOU; ZENGER, 2008). Lee (2001), Gottschalk (2006), Lacity et al. (2010), entre outros, argumentam que o compartilhamento do conhecimento é fundamental para o sucesso da terceirização de TI.

\subsection{Compartilhamento do Conhecimento}

Nonaka e Takeuchi (1995) definem o conhecimento como informação combinada com experiência, outros conhecimentos, crenças, as lições aprendidas e assimiladas por pessoas. Estudos anteriores indicaram que o conhecimento pode ser classificado em dois tipos: conhecimento explícito e conhecimento tácito (POLANYI, 1983; NONAKA, 1994; ALAVI; LEIDNER, 2001; WILLIAMS, 2006). A dimensão de conhecimento tácito ou conhecimento explícito reflete com a variabilidade da natureza intangível de conhecimento (NONAKA; TAKEUCHI, 1995). Ao interagir e compartilhar conhecimento tácito 
Roberta Rodrigues Faoro, Mírian Oliveira, Marcelo Faoro de Abreu O compartilhamento do conhecimento e a inovação nos relacionamentos interorganizacionais do tipo terceirização de tecnologia da informação

e explícito com outros, o indivíduo melhora a capacidade de definir uma situação ou problema, e aplica o seu conhecimento, de modo a atuar e, especificamente, de resolver o problema (NONAKA; VON KROGH; VOELPEL, 2006).

Sato et al. (2015, p. 321) concluem que,

o conhecimento é ainda percebido como poder, a linguagem muitas vezes não consensual entre os especialistas, principalmente com relação aos conceitos, a resistência a novas ideias, a acomodação de alguns participantes que se isolavam devido à sobreposição de alguns sobre os outros e a limitação do tempo devido à extensão dos debates.

As pesquisas de gestão do conhecimento, muitas vezes, giram em torno da criação e compartilhamento de conhecimento dentro de uma organização em particular (ARGOTE, 1999; NONAKA, 1994; NONAKA; TAKEUCHI, 1995). No entanto, outra perspectiva enfatiza o estudo de compartilhamento de conhecimento através das fronteiras organizacionais (DYER; NOBEOKA, 2000; OWEN-SMITH; POWELL, 2004; POWELL; BRANTLEY, 1992). Dutta (2012) descreve que o relacionamento interorganizacional é qualquer acordo formal ou semiformal entre duas ou mais empresas, que fornece a cada participante um benefício da colaboração que não está disponível ou é proibitivamente caro para adquirir no mercado ou desenvolver na própria empresa.

Além disso, o compartilhamento de conhecimento interorganizacional é uma das principais fontes para inovação (PARDO et al., 2006). Portanto, as organizações que estão pensando em entrar ou estabelecer um ambiente de compartilhamento de conhecimento com seus parceiros de negócios devem inevitavelmente refletir sobre a seguinte questão: Os benefícios superam os riscos? (SOPER; DEMIRKAN; GOUL, 2007).

Por um lado, um ambiente de compartilhamento de conhecimento interorganizacional permite às organizações participantes ampliarem seu capital intelectual, obtendo novos ativos de conhecimentos (HARDY; PHILLIPS; LAWRENCE, 2003), "os pilares da economia moderna” (SOPER et al., 2007, p. 
Roberta Rodrigues Faoro, Mírian Oliveira, Marcelo Faoro de Abreu O compartilhamento do conhecimento e a inovação nos relacionamentos interorganizacionais do tipo terceirização de tecnologia da informação

470). Por outro lado, uma organização que compartilha os seus ativos de conhecimento fora dos limites da empresa, se expõe à possibilidade de que os ativos de conhecimento venham a ser adquiridos por um destinatário nãosancionado (por exemplo, um concorrente direto), erodindo, assim, a posição competitiva da organização de origem. Decidir se deve ou não participar de um ambiente de compartilhamento de conhecimento interorganizacional é, pois, de importância fundamental para muitas organizações (SOPER et al., 2007, p. 470).

Neste sentido, os resultados de Bandyopadhyay e Pathak (2007) apresentam implicações importantes para o sucesso dos projetos de terceirização. Quando uma empresa terceiriza, a fim de beneficiar-se de um conjunto de competências complementares, a administração da empresa terá de estar envolvida não só na negociação do contrato de terceirização, mas também nas modalidades de funcionamento e na interação entre as duas empresas. Uma vez que os funcionários das duas empresas provavelmente seriam mutuamente antagônicos, a administração teria de estabelecer as diretrizes de interação entre as empresas. Estas diretrizes podem incluir detalhes como o número de horas que os funcionários teriam de gastar uns com os outros, para compartilhar o conhecimento um do outro (e, simultaneamente, definir-se um controle efetivo dessa interação), a frequência de reuniões entre os gestores, etc. Tais diretrizes mutuamente acordadas e o grau de compartilhamento de conhecimentos resultarão em melhores retornos para os funcionários e, por extensão, para a empresa.

O compartilhamento de conhecimento pode ajudar as organizações a melhorarem a sua capacidade de reagir à incerteza e complexidade do ambiente (PARDO et al., 2006). O compartilhamento de conhecimento pode ser um resultado da educação formal e de mecanismos sociais informais. $O$ primeiro pode ser por feiras de tecnologia, reuniões ou pedidos de informações, enquanto que os mecanismos informais podem girar em torno de aferição conjunta de pesquisa de fornecedores, engenharia de visitas às instalações ou demonstrações de produtos, entre outros (MONCZKA; TRENT; HANDFIELD, 
Roberta Rodrigues Faoro, Mírian Oliveira, Marcelo Faoro de Abreu O compartilhamento do conhecimento e a inovação nos relacionamentos interorganizacionais do tipo terceirização de tecnologia da informação

2000). Em um ambiente em que as empresas estão confiando cada vez mais em desenvolvimento de equipes interorganizacionais (MCDONOUGH; KAHN; BARCZAK, 2001), os mecanismos de socialização podem ser uma fonte importante para o compartilhamento de conhecimentos e para superação das barreiras comportamentais e culturais nestas estruturas de projetos. Sendo assim, o compartilhamento de conhecimento, dentro das equipes interorganizacionais, melhora a contribuição do fornecedor para os resultados do desenvolvimento do produto (LAWSON et al., 2009).

Desta forma, estudos têm sido feitos para encontrar abordagens e mecanismos para aumentar o compartilhamento do conhecimento (WILLEM; BUELENS, 2009). Há alguns estudos sobre mecanismos tecnológicos (ALAVl; LEIDNER, 2001; SHER; LEE, 2004), outros têm procurado estimular os indivíduos a compartilhar conhecimento por meio dos sistemas de incentivos (BOCK; LEE; ZMU, 2005; HSU, 2006; HWANG; KIM, 2007). Já o estudo de Lynskey (1999) destaca a importância dos mecanismos formais e informais. Os mecanismos formais, como consultoria, documentação, treinamento e demonstrações são adequados para o compartilhamento de conhecimento explícito, enquanto abordagens tais como reuniões "face-a-face", destaque de funcionários, e interações em primeira mão são necessárias para compartilhar o conhecimento tácito.

Embora as relações ou interações formais, incluindo programas de treinamento e equipes de trabalhos estruturados, desempenham um papel importante no sentido de facilitar o compartilhamento de conhecimento dos funcionários, Truran (1998) constatou que a maior quantidade de conhecimento é compartilhada em interações informais. Associado a isso, o estudo de Janowicz-Panjaitan e Noorderhaven (2008) conclui que embora os mecanismos formais e informais promovam a aprendizagem interorganizacional, o excesso de formalização impede o aprendizado.

Além disso, pesquisadores têm enfatizado a importância da TI para o compartilhamento do conhecimento (ALAVI; LEIDNER, 2001; TEECE, 2001; DAVENPORT, 1997; GRANT, 1996). Para Alavi e Leidner (2001) os 
Roberta Rodrigues Faoro, Mírian Oliveira, Marcelo Faoro de Abreu O compartilhamento do conhecimento e a inovação nos relacionamentos interorganizacionais do tipo terceirização de tecnologia da informação

mecanismos tecnológicos podem aumentar o compartilhamento do conhecimento. Por exemplo, as redes de computadores, painéis eletrônicos e grupos de discussão facilitam o contato entre aqueles que buscam conhecimento e aqueles que controlam o acesso ao conhecimento. Davis e Riggs (1999) estendem a lista de aplicações de TI para o compartilhamento de conhecimento para incluir sistemas de internet baseados em rede, sistemas de groupware, intranets, bancos de dados, sistemas de gerenciamento de dados eletrônicos e sistemas de informação de gestão do conhecimento. King (1999) também indica que a concepção e fornecimento de um sistema de gestão do conhecimento que aborda precisamente as necessidades do usuário é um dos fatores mais importantes que afetam o sucesso do compartilhamento de conhecimento.

Portanto, os mecanismos de socialização podem desempenhar um papel importante no sentido de facilitar o compartilhamento do conhecimento pelo caminho das fronteiras organizacionais. Ao fornecer uma "linguagem comum" e criar entendimento compartilhado, os mecanismos de socialização facilitam o compartilhamento tanto do conhecimento explícito quanto do conhecimento tácito entre os parceiros (LAWSON et al., 2009).

\subsection{Inovação}

A inovação pode ser vista como uma fonte de criação de valor (DAMANPOUR; GOPALAKRISHNAN, 1998). A inovação é considerada um importante tópico de pesquisa em várias disciplinas como economia, gestão, marketing, tecnologia, engenharia e estudos das organizações (BAREGHEH; ROWLEY; SAMBROOK, 2009). No entanto, não se pode falar de inovação sem citar Joseph Schumpeter, pois, somente a partir do seu trabalho - "Teoria do Desenvolvimento Econômico, 1934", estabeleceu-se uma relação entre inovação e desenvolvimento econômico, pois o estudo aborda a teoria da "destruição criativa", sustentando que o sistema capitalista progride por revolucionar constantemente sua estrutura econômica: novas firmas, novas 
Roberta Rodrigues Faoro, Mírian Oliveira, Marcelo Faoro de Abreu O compartilhamento do conhecimento e a inovação nos relacionamentos interorganizacionais do tipo terceirização de tecnologia da informação

tecnologias e novos produtos substituem constantemente os antigos (SCHUMPETER, 1982).

As organizações devem inovar, a fim de renovar o valor de seus ativos (SCHUMPETER, 1950). Brozen (1951) afirma que a inovação, bem como a invenção e a imitação, consistem em atividades de mudanças tecnológicas que conduzem ao crescimento econômico. Thompson's (1965, p. 2) define inovação como "a geração, aceitação e implementação de novas ideias, produtos, processos ou serviços". Drucker (1969) acredita que a inovação empresarial seria uma parte fundamental das atividades gerenciais. Nelson e Winter (1982) sugerem que a inovação, é um dos fatores principais da empresa, que desencadeia mudanças econômicas. Para Ziviani, Ferreira e Neves (2015, p. 162) "a inovação é um fenômeno enraizado em conhecimento e, particularmente, em informação. Identificar onde as empresas buscam ideias para inovar pode ser um importante sinalizador para a compreensão de aspectos de sua dinâmica inovativa". Assim, a inovação tem sido um dos principais temas de gestão para os estudiosos das organizações. Popadiuk e Choo (2006) acreditam que se uma ideia não foi desenvolvida e transformada num produto, processo ou serviço, ou se não tiver sido comercializada, então não seria classificada como uma inovação.

Neste sentido, Zaninelli entende que,

a inovação é imprescindível para que as empresas possam obter vantagens sustentáveis perante seus concorrentes e se manterem competitivas num mercado que se encontra em constante mutação. Para tal, num primeiro momento, as empresas devem ter um entendimento e uma predisposição para melhorar continuamente as suas atividades e processos, para que posteriormente possam empregar esforços visando à transformação das oportunidades em realidade (ZANINELLI, 2012, p.137).

Outras variações na definição de inovação surgem de diferentes perspectivas disciplinares. Por exemplo, na gestão do conhecimento, o foco é o conhecimento, vital para a inovação ou até mesmo um tipo de inovação. Como argumenta Plessis (2007, p 21), "inovação como a criação de novos 
Roberta Rodrigues Faoro, Mírian Oliveira, Marcelo Faoro de Abreu O compartilhamento do conhecimento e a inovação nos relacionamentos interorganizacionais do tipo terceirização de tecnologia da informação

conhecimentos e ideias para facilitar novos resultados de negócios, visando à melhoria dos processos internos do negócio e estruturas e criar produtos e serviços orientados para o mercado". Já em definições relacionadas às tecnologias, o foco principal da inovação deve ser um produto relacionado às novas tecnologias (NORD; TUCKER, 1987).

Se as organizações podem gerenciar as mudanças para se tornarem inovadoras, então devem ter um ambiente que promova a inovação (ORANGE et al., 2007). Neste sentido, tem-se a taxonomia, sugerida por Schumpeter (1934): em primeiro lugar, diferenciar os tipos de inovação com base no objeto da mudança, ou seja, inovação de produto, inovação de processo, inovação de marketing e inovação organizacional (OSLO, 2005). Em segundo lugar, podese tentar fazer a diferença entre as inovações com base em sua "novidade", ou seja, com base na extensão da mudança (VARIS; LITTUNEN, 2010), ou seja, inovação incremental e inovação radical (SCHUMPETER, 1982).

Para Schumpeter (1934), as inovações radicais são aquelas que provocam grandes mudanças no mundo, enquanto as inovações incrementais promovem o processo de mudança continuamente. Para Garcia e Calantone (2002), não há dúvida de que nem todas as inovações são a mesma coisa. Inovações incrementais são pequenas alterações e modificações em produtos e tecnologias, enquanto que inovações radicais representam a base para o desenvolvimento de novos produtos e serviços (GARCIA; CALANTONE, 2002).

\section{PROCEDIMENTOS METODOLÓGICOS}

A pesquisa aqui apresentada é de natureza qualitativa. $O$ método qualitativo refere-se a "um conjunto de técnicas interpretativas que procuram descrever, decodificar, traduzir, e de outra forma chegar a um acordo com o significado, não com a frequência, é claro, mais ou menos fenômenos que ocorrem naturalmente no mundo social" (VAN MAANEN, 1979, p. 520).

A estratégia de pesquisa adotada neste trabalho baseia-se em estudos de casos. O estudo de caso "envolve o estudo de um tema explorado através 
Roberta Rodrigues Faoro, Mírian Oliveira, Marcelo Faoro de Abreu O compartilhamento do conhecimento e a inovação nos relacionamentos interorganizacionais do tipo terceirização de tecnologia da informação

de um ou mais casos dentro de um sistema limitado" (CRESWELL, 2007, p. 73). O estudo de caso foi exploratório. A pesquisa exploratória busca descrever de forma objetiva e direta "eventos" e "fatos" de interesse (DUBÉ; PARÉ, 2003). Além disso, Merriam (1998) sugere que a inclusão de vários casos em um estudo faz os resultados e interpretações mais convincentes. Portanto, a lógica por trás da escolha de um estudo de múltiplos casos, em vez de um único estudo de caso, foi conduzida pela posição que o estudo de múltiplos casos de um mesmo fenômeno pode corroborar, qualificar ou estender os resultados.

Neste sentido, esta pesquisa é um estudo de múltiplos casos (MERRIAM, 1998), sendo que os casos deverão ter relacionamentos interorganizacionais do tipo terceirização de ERPs. A escolha desta área para estudo baseia-se no fato de que muitas empresas estão utilizando sistemas de ERP para cortar custos, padronizar as operações e alavancar processos comuns em toda a organização. ERPs permitem que as empresas tenham visões mais convergentes de suas informações através da integração de processos, utilizando um banco de dados centralizado e conjuntos integrados de módulos de softwares (SCOTT; KAINDL, 2000). Assim sendo, o primeiro critério para seleção dos casos é que fossem formados por duas ou mais empresas envolvidas em terceirização de ERP.

Outro critério para a seleção dos casos foi a diversidade de segmentos de atuação das empresas clientes de ERPs para, com isso, tentar evitar a contaminação pelas características especiais de cada segmento de atuação, ou seja, buscou-se reduzir algum eventual viés relacionado aos segmentos de atuação das empresas. Associado a isso, o último critério utilizado para a seleção das empresas clientes de ERPs foi a existência de alguma dependência destas empresas em relação ao software ERP para a implementação de suas estratégias ou operações.

As fontes de informações desta pesquisa foram: entrevistas semiestruturadas, documentos de interesse da pesquisa, tais como sites, organograma, manuais, documentos sobre política da empresa, código de 
Roberta Rodrigues Faoro, Mírian Oliveira, Marcelo Faoro de Abreu O compartilhamento do conhecimento e a inovação nos relacionamentos interorganizacionais do tipo terceirização de tecnologia da informação

conduta e ética, entre outros, além da observação direta. As entrevistas, as quais tiveram em média a duração de uma hora, foram gravadas e posteriormente transcritas. A adoção de mais de uma forma de coleta de dados visou à triangulação (DUBÉ; PARÉ, 2003), o que oferece oportunidades para o pesquisador buscar a convergência dos resultados e observar a sua sobreposição, caso ocorra, e as diferentes facetas de um fenômeno que surgem de várias fontes (CRESWELL, 2007). Desta forma, a observação direta nas empresas, assim como a pesquisa documental, ajuda na complementação das informações coletadas em um estudo de caso (TRIVIÑOS, 1987).

A análise dos dados coletados foi realizada através da análise de conteúdo temática. A análise de conteúdo constitui uma técnica que trabalha os dados coletados, objetivando a identificação do que está sendo dito a respeito de um determinado tema (VERGARA, 2005). A análise de conteúdo seguiu as três etapas da técnica de Bardin (2006): 1) pré-análise, 2) exploração do material, 3) tratamento dos resultados, inferência e interpretação.

Com a intenção de sanar eventuais problemas na realização da pesquisa, foram tomados alguns cuidados e procedimentos que aumentam a validade e a confiabilidade da pesquisa. Foram utilizados os critérios de validade do constructo, validade externa e confiabilidade. A validade interna não foi utilizada, pois não se aplica, uma vez que esta pesquisa é exploratória e a validade interna, segundo Yin (2001), é específica para estudos explanatórios ou causais.

\section{ANÁLISE DOS ESTUDOS DE CASO}

Nesta seção, apresenta-se a análise dos casos. No primeiro caso, os nomes das empresas foram substituídos respectivamente por Alpha (fornecedora), CA1 e CA2 (clientes) com o objetivo de preservar informações estratégicas e confidenciais das mesmas. Nos demais casos (caso 2, caso 3 e caso 4), as empresas fornecedoras de TI estudadas foram: Domper Consultoria e Sistemas, NL Informática e Totvs/RS. Os nomes das empresas clientes foram 
Roberta Rodrigues Faoro, Mírian Oliveira, Marcelo Faoro de Abreu O compartilhamento do conhecimento e a inovação nos relacionamentos interorganizacionais do tipo terceirização de tecnologia da informação

substituídos respectivamente por CD1 (Cliente Domper 1), CD2 (Cliente Domper 2), CNL1 (Cliente NL 1), CNL2 (Cliente NL 2), CTotvs1 (Cliente Totvs 1), e CTotvs 2 (Cliente Totvs 2), com o objetivo de preservar informações estratégicas e confidenciais das mesmas.

A análise aborda o compartilhamento do conhecimento entre empresas clientes e a empresa fornecedora do ERP nas relações interorganizacionais do tipo terceirização de TI, assim como a inovação, com base nas percepções dos entrevistados. A análise foi estruturada em duas partes: descrição das empresas (4.1) e análises dos dados sobre compartilhamento do conhecimento e inovação (4.2).

\subsection{Descrição das Empresas}

No Quadro 1 são apresentadas as características gerais das empresas dos estudos de casos: 1, 2, 3 e 4. Considerando que o porte da empresa foi classificado com base na sua Receita Operacional Bruta (ROB) anual, conforme BNDES (2011).

Quadro 1 - Características Gerais das Empresas Estudadas

\begin{tabular}{|c|c|c|c|}
\hline \multicolumn{4}{|c|}{ ESTUDO DE CASO 1} \\
\hline CARACTERÍSTICAS & ALPHA & CA1 & CA2 \\
\hline $\begin{array}{l}\text { Tempo de } \\
\text { Funcionamento }\end{array}$ & 18 anos & 23 anos & 6 anos \\
\hline $\begin{array}{l}\text { Porte da Empresa } \\
\text { (ROB) }\end{array}$ & Pequena & Média & Pequena \\
\hline Ramo de Atividade & Serviços de TI & Comércio e Indústria & Comércio \\
\hline $\mathrm{N}^{\circ}$ de Colaboradores & 10 & 35 & 14 \\
\hline $\begin{array}{l}\text { Estrutura } \\
\text { Organizacional }\end{array}$ & Equipe & Funcional & Linear \\
\hline \multirow{2}{*}{\multicolumn{2}{|c|}{ Tempo de Parceria com o Fornecedor (Alpha) }} & 3 anos & 4 anos \\
\hline & & \multicolumn{2}{|c|}{ ESTUDO DE CASO 2} \\
\hline CARACTERÍSTICAS & DOMPER & CD1 & CD2 \\
\hline $\begin{array}{l}\text { Tempo de } \\
\text { Funcionamento }\end{array}$ & 17 anos & 28 anos & 27 anos \\
\hline $\begin{array}{l}\text { Porte da Empresa } \\
\text { (ROB) }\end{array}$ & Pequena & Média & Média \\
\hline Ramo de Atividade & Serviços de TI & Agronegócios & Agronegócios \\
\hline $\mathrm{N}^{\circ}$ de Colaboradores & 17 & 260 & 200 \\
\hline $\begin{array}{l}\text { Estrutura } \\
\text { Organizacional }\end{array}$ & Projetos & Funcional & Funcional \\
\hline \multicolumn{2}{|c|}{ Tempo de Parceria com o Fornecedor (Domper) } & 10 anos & 12 anos \\
\hline
\end{tabular}


Roberta Rodrigues Faoro, Mírian Oliveira, Marcelo Faoro de Abreu O compartilhamento do conhecimento e a inovação nos relacionamentos interorganizacionais do tipo terceirização de tecnologia da informação

\begin{tabular}{|l|l|l|l|}
\hline CARACTERÍSTICAS & NL & CNL1 & CNL2 \\
\hline $\begin{array}{l}\text { Tempo de } \\
\text { Funcionamento }\end{array}$ & 33 anos & 61 anos & 28 anos \\
\hline $\begin{array}{l}\text { Porte da Empresa } \\
\text { (ROB) }\end{array}$ & Média & Grande & Grande \\
\hline Ramo de Atividade & Serviços de TI & Comércio e Indústria & Comércio \\
\hline No de Colaboradores $^{\circ}$ & 100 & 150 & 1450 \\
\hline $\begin{array}{l}\text { Estrutura } \\
\text { Organizacional }\end{array}$ & Funcional & Funcional & Funcional \\
\hline Tempo de Parceria com o Fornecedor (NL) & 10 anos & 9 anos \\
\hline \multicolumn{2}{|l|}{ ESTUDO DE CASO 4 } & DTOTVS2 \\
\hline CARACTERíSTICAS & TOTVS/RS & CTOTVS1 & 75 anos \\
\hline $\begin{array}{l}\text { Tempo de } \\
\text { Funcionamento }\end{array}$ & 7 anos & 25 anos & Grande \\
\hline $\begin{array}{l}\text { Porte da Empresa } \\
\text { ROB) }\end{array}$ & Média & Média & Comércio e ServiçOS \\
\hline Ramo de Atividade & Serviços de TI & Indústria & 1300 \\
\hline No de Colaboradores & 350 & 100 & Funcional \\
\hline $\begin{array}{l}\text { Estrutura } \\
\text { Organizacional }\end{array}$ & Funcional & Funcional & 10 anos \\
\hline Tempo de Parceria com o Fornecedor (TOTVS) & 7 anos & \\
\hline
\end{tabular}

Fonte: Dados da Pesquisa

Em relação as empresas fornecedoras de $\mathrm{Tl}$, pode perceber que a empresa Alpha presta serviços de TI para as empresas dos ramos de comércio e indústria. A empresa é estruturada por equipes, lideradas pelos dois sócios, que são basicamente os dois gestores da Alpha, sendo que um dos sócios responde pela equipe de software e o outro sócio responde pela equipe de hardware. Os demais setores como comercial, atendimento e financeiro atendem as duas áreas de empresa, ou seja, a área de software e a área de hardware.

Já a empresa Domper atua no desenvolvimento de softwares e consultorias em tecnologia da informação para as empresas dos ramos de agronegócios, comércio, indústria, logística e serviços. Com sede em Vacaria/RS, a empresa atende todo o território nacional, sendo que os seus principais clientes estão localizados nos estados do Rio Grande do Sul, Santa Catarina, São Paulo, Bahia e Mato Grosso do Sul. A empresa é dividida por duas gerências: a gerência administrativa, comercial e financeira e a gerência de projetos, sendo que, a gerência de projetos é dividida pelas áreas de negócios que a empresa atua, ou seja, agronegócios, comércio, indústria, 
Roberta Rodrigues Faoro, Mírian Oliveira, Marcelo Faoro de Abreu O compartilhamento do conhecimento e a inovação nos relacionamentos interorganizacionais do tipo terceirização de tecnologia da informação

logística e serviços. A empresa ainda possui três revendas: uma em Flores da Cunha/RS, uma em Caxias do Sul/RS e uma em Salvador/BA. O mercado onde a empresa atua é composto, principalmente, por empresas de porte médio nos diferentes setores da economia, entretanto, destaca-se o setor de fruticultura, onde detêm uma participação aproximadamente de $70 \%$ do mercado nacional de softwares para a gestão da produção, comercialização, armazenagem e processamento de maçãs, sendo assim, somente o atendimento do ERP das empresas de fruticultura é 100\% realizado pela Domper. Neste sentido, a escolha das empresas clientes para o estudo foi do setor de fruticultura, pois todo o ciclo de prestação de serviços: projeto, implementação, configuração implantação e manutenção é realizado pela empresa Domper. As empresas clientes estudadas estão localizadas na região Nordeste do estado do Rio Grande do Sul.

A NL presta serviços de TI para as empresas dos ramos de comércio, indústria e serviços. Com sede em Caxias do Sul /RS, escritório em São Paulo/SP, uma franquia na região do Alto Taquari/RS e atendendo clientes em todo o país. A NL conta com uma vasta carteira de clientes, tais como Lojas Marisa, Grupo Grazziotin, Converplast, Top Internacional, Lojas Esplanada, Sodexo|Puras, entre outros. A estrutura organizacional da NL é composta por um Conselho de Sócios, ou seja, a diretoria da empresa, abaixo tem duas áreas de assessorias (uma na área de $\mathrm{RH}$ e qualidade e a outra na área técnica, que é exercida por uma das sócias) e quatro gerências (gerência comercial de marketing, gerência de tecnologia, gerência de consultoria e gerência administrativa-financeira). Segundo o Diretor, a empresa possui em torno de $1,1 \%$ do mercado nacional de $\mathrm{TI}$, sendo que, o maior foco da empresa está no segmento de varejo. Neste sentido, a escolha das empresas clientes para o estudo foi do ramo do comércio. As empresas clientes estudadas estão localizadas na região Nordeste do estado do Rio Grande do Sul.

Por fim, a matriz da franquia do RS está localizada em Porto Alegre e possui uma filial em Caxias do Sul e estão constituindo uma filial em Santa Maria. O objetivo da franquia RS é comercializar produtos, soluções e serviços 
Roberta Rodrigues Faoro, Mírian Oliveira, Marcelo Faoro de Abreu O compartilhamento do conhecimento e a inovação nos relacionamentos interorganizacionais do tipo terceirização de tecnologia da informação

da Totvs e prover serviços de implementação, consultoria, treinamentos fazendo com que os clientes utilizem melhor os sistemas para o desenvolvimento do seu negócio. Ainda a franquia RS tem uma pequena fábrica de software que desenvolve algumas personalizações, mas toda parte de construção de soluções Totvs relacionado à construção dos programas, não é responsabilidade das franquias. Em relação à estrutura organizacional na franquia Totvs Rio Grande do Sul é formada por um executivo CEO que atende todo estado, responde por toda operação da empresa, mais dois diretores regionais, um para a Serra Gaúcha e um para Santa Maria. Esses diretores operacionais, eles respondem tanto pela parte comercial quanto pela prestação de serviço, que por sua vez tem gestores abaixo deles, tanto comerciais quanto de serviços. Existe em cada unidade dois gestores comerciais, um para atender clientes base e outro para prospectar novos negócios. E existe um gestor geral de serviços também com sua estrutura de apoio, um para cada unidade, um para Porto Alegre e um para Caxias do Sul. Possui também uma área administrativa financeira e uma área de marketing, que são ligadas diretas ao presidente, ao CEO, que atende globalmente as duas regionais, tanto Porto Alegre quanto Serra Gaúcha. As empresas clientes estudadas estão localizadas na região Nordeste do estado do Rio Grande do Sul.

\subsection{Comparação e Discussão dos Resultados dos Casos}

Os dois principais motivos para a terceirização da TI encontrados nos casos foram a redução de custos e a priorização do foco no negócio, como pode ser observado na Figura 1. 
Roberta Rodrigues Faoro, Mírian Oliveira, Marcelo Faoro de Abreu O compartilhamento do conhecimento e a inovação nos relacionamentos interorganizacionais do tipo terceirização de tecnologia da informação

Figura 1 - Motivos para a Terceirização de TI x Empresas

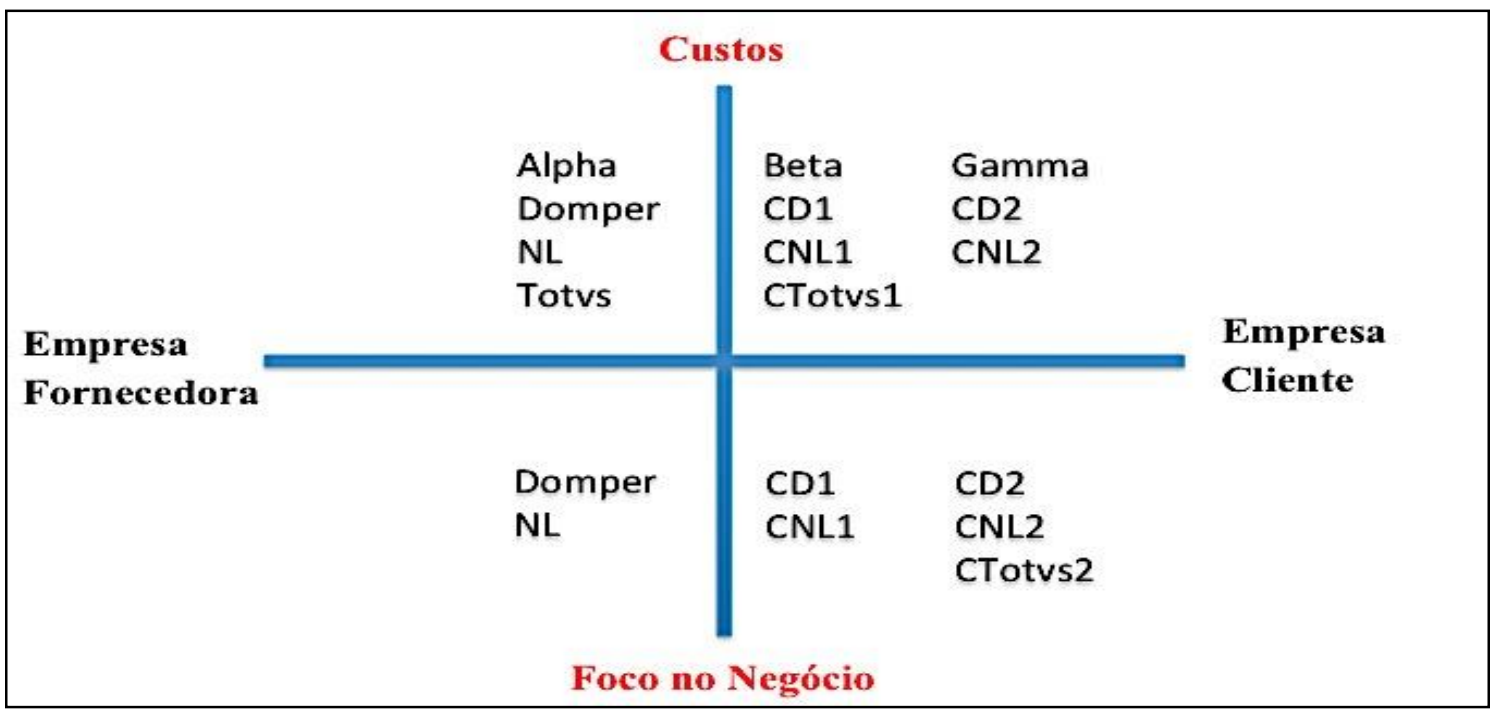

Fonte: Elaborado pelos Autores

A decisão de "fazer ou comprar" no contexto da TI tem uma rica base teórica, especialmente em relação à teoria dos custos de transação (CHADEE; RAMAN, 2009). Malone et al. (1987, p. 485) argumentam que transações mais eficientes ajudam as empresas a reduzir os custos de coordenação, que são definidos como os custos de transação de todas as informações necessárias para o processamento de coordenar o trabalho de pessoas e máquinas, que executam processos primários e "levar em conta os custos de coleta de informação, negociação de contratos e proteger contra os riscos de negociação oportunista", o que está alinhado com os resultados identificados nos casos estudados nesta pesquisa.

Sendo assim, percebe-se, uma forte tendência de terceirização de TI, pois está sendo desenvolvida em todo o mundo com uma velocidade notável e em várias formas (QI; CHAU, 2012). Logo, o novo desafio reside principalmente na forma de transferência do conhecimento entre o fornecedor e o cliente. Conhecimentos, que anteriormente poderiam ser transferidos entre pessoas da mesma organização, agora têm que ser transferidos através de duas ou mais organizações diferentes. Consequentemente, os canais para a 
Roberta Rodrigues Faoro, Mírian Oliveira, Marcelo Faoro de Abreu O compartilhamento do conhecimento e a inovação nos relacionamentos interorganizacionais do tipo terceirização de tecnologia da informação

transferência de conhecimento serão mais formais e impessoais na terceirização (GOTTSCHALK, 2006).

Consonante ao compartilhamento do conhecimento foi possível perceber que a troca de conhecimentos tácitos e explícitos ocorre tanto nas empresas clientes quanto nas empresas fornecedoras, a diferença é que as empresas fornecedoras (Alpha, Domper, NL e Totvs) dão maior ênfase na formalização do conhecimento compartilhado, ou seja, "tentam transformar todo" o conhecimento tácito compartilhado com seus clientes em conhecimento explícito, pois as mesmas documentam todos atendimentos e armazenam em softwares, com o objetivo de ter melhor gerenciamento dos clientes. Dizendo de outra forma, as empresas fornecedoras de ERPs têm como prioridade a codificação do conhecimento, ou seja, a conversão do nível tácito para o explícito (NONAKA; TAKEUCHI, 1995). Neste sentido, os principais mecanismos formais citados pelas empresas tanto clientes quanto fornecedoras foram: treinamentos, reuniões e consultorias. Sendo assim, pôdese evidenciar o maior interesse das empresas fornecedoras em explicitar o conhecimento compartilhado com as empresas clientes, um dos motivos percebidos no estudo, foi que as fornecedoras têm interesses em conhecerem melhor os seus clientes e com isso, melhorar o seu produto, pois o produto da fornecedora é o ERP, em outras palavras, quanto mais conhecimentos as empresas fornecedoras compartilharem com seus clientes, mais vão conseguir entender seus clientes e desenvolverem melhorias em seus ERPs.

Além disso, as empresas clientes formalizam os seus conhecimentos quando solicitados pelas empresas fornecedoras, como exemplo o e-mail ou help desk mas, por outro lado, pôde-se inferir que maioria das empresas clientes, preferem as conversas informais por meio de ligações telefônicas, skype ou nas visitas realizadas por iniciativa dos profissionais das fornecedoras para compartilharem seus conhecimentos. Com exceção das empresas Clientes 2 dos casos 3 e 4, pois estas duas empresas possuem um setor de $\mathrm{TI}$, os quais têm como responsabilidade gerenciar os contratos terceirizados da $\mathrm{TI}$, logo, é necessário que os setores de TI das empresas Clientes 2 dos casos 3 e 
Roberta Rodrigues Faoro, Mírian Oliveira, Marcelo Faoro de Abreu O compartilhamento do conhecimento e a inovação nos relacionamentos interorganizacionais do tipo terceirização de tecnologia da informação

4, tenham as documentações armazenadas dos serviços realizados pelas empresas fornecedoras. Destaca-se aqui, o resultado sobre as empresas clientes, cuja maioria prefere compartilhar informalmente os conhecimentos com a fornecedora. Este resultado converge com o estudo de Truran (1998), o qual entende a importância das relações ou interações formais como, por exemplo, programas de treinamento e equipes de trabalhos estruturados, no sentido de facilitar o compartilhamento de conhecimento dos funcionários, por outro lado, o autor, constatou que a maior quantidade de conhecimento é compartilhada em interações informais. Associado a isso, o estudo de Janowicz-Panjaitan e Noorderhaven (2008) conclui que embora os mecanismos formais e informais promovam a aprendizagem interorganizacional, o excesso de formalização impede o aprendizado.

Os resultados encontrados em relação aos tipos de conhecimentos são ilustrados na Figura 2. Ainda assim, um dos motivos percebidos entre as empresas clientes em relação a formalização do conhecimento é a "terceirização da responsabilidade", dizendo de outra forma, com exceção das duas empresas Clientes 2 (casos 3 e 4), as demais comentaram que quando precisam de alguma documentação podem solicitar para a empresa fornecedora, pois as mesmas sabem que as empresas fornecedoras formalizam todos os atendimentos, serviços, enfim, todo o conhecimento que é compartilhado através da terceirização de ERP.

Figura 2 - Tipos de Conhecimentos x Empresa

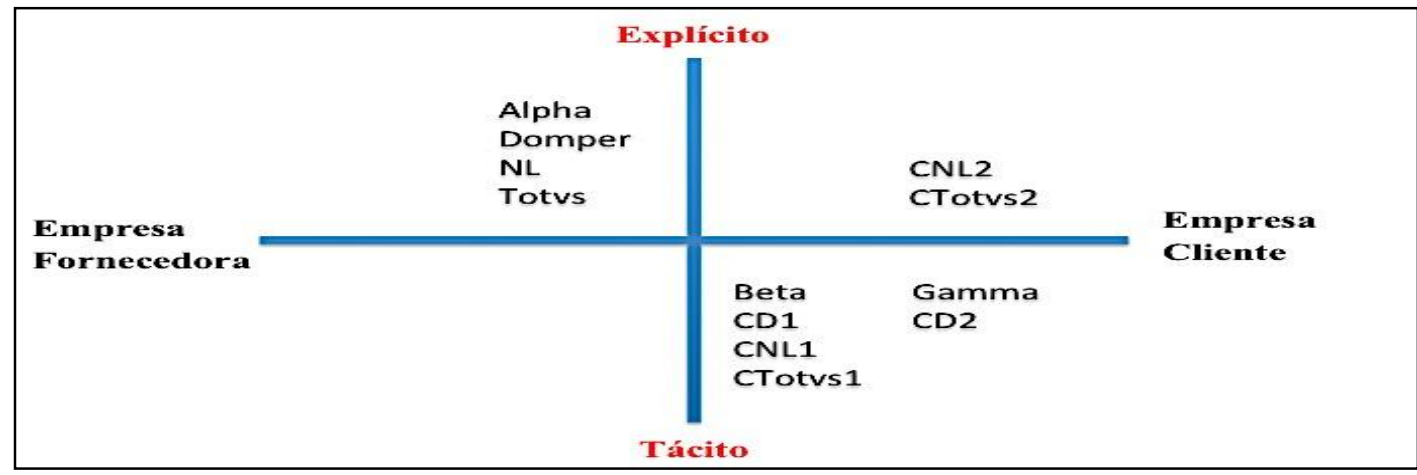

Fonte: Elaborado pelos Autores

Inf. Inf., Londrina, v. 22, n. 1, p. 263 - 292, jan./abr., 2017. http:www.uel.br/revistas/informacao/ 
Roberta Rodrigues Faoro, Mírian Oliveira, Marcelo Faoro de Abreu O compartilhamento do conhecimento e a inovação nos relacionamentos interorganizacionais do tipo terceirização de tecnologia da informação

Por conseguinte, foi possível perceber em todas as empresas da pesquisa, a existência de inovações através da terceirização do ERP, como pode ser observado na Figura 3. Entretanto, observou-se que as empresas fornecedoras são as que mais inovam, juntamente com a empresa Cliente 2 da Totvs (caso 4). Ou seja, as empresas mencionadas anteriormente, inovaram nos quatro tipos de inovações, ou seja, inovação nos produtos, nos processos, no marketing e na gestão organizacional. Este resultado ratifica os achados anteriores (compartilhamento do conhecimento), os quais apresentaram estas empresas, formalizando os conhecimentos compartilhados com o objetivo de desenvolver, explorar e inovar seus produtos e serviços.

Outro ponto observado, foi a inovação de processos presentes em todas as empresas clientes, logo, pôde-se inferir que em ambientes de terceirização do ERP as empresas clientes, obtiveram melhorias significativas nos seus processos: agilidade, controle e integração dos processos. Ainda, pôde-se concluir que as empresas que mais inovam com referência aos tipos de inovação são as clientes da Totvs, a empresa Cliente 2 e a empresa Cliente 1 respectivamente. Isto pode ser explicado, baseado no posicionamento da Totvs, ou seja, a Totvs tem como objetivo a inovação, com isso, os clientes que conseguirem compartilhar conhecimentos com a fornecedora têm grandes possibilidades de inovar. Ainda assim, pôde-se perceber nas empresas clientes, inovações de marketing (Cliente 1 da Domper e da Totvs e Cliente 2 da NL) e inovações organizacionais (Cliente 2 da Domper e Cliente 1 da Totvs).

Figura 3 - Tipos de Inovação x Empresa

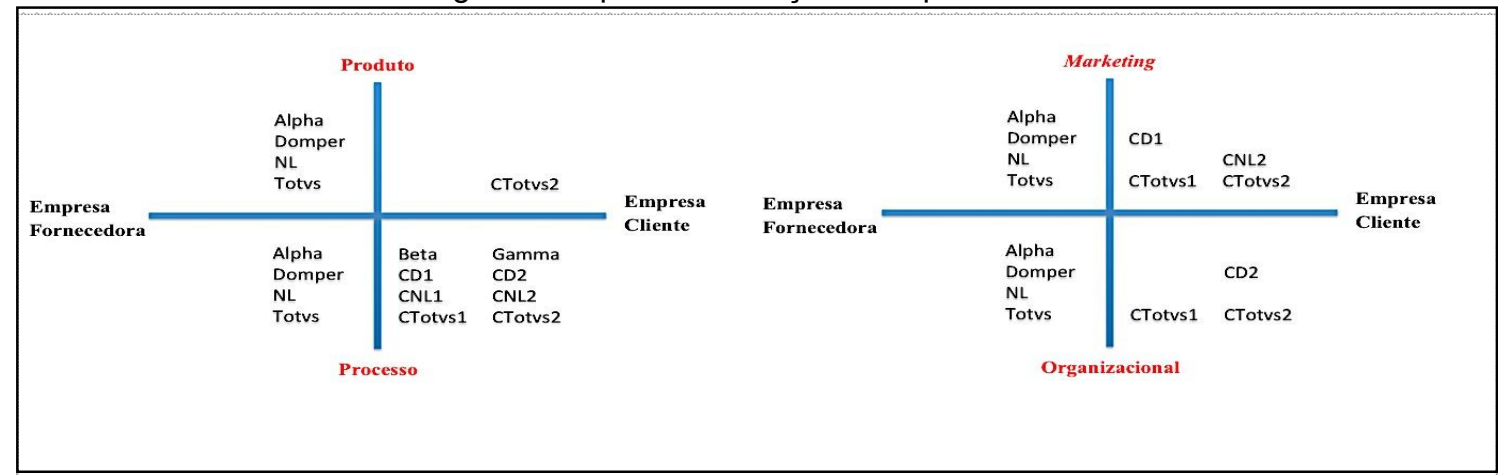

Fonte: Elaborado pelos Autores

Inf. Inf., Londrina, v. 22, n. 1, p. 263 - 292, jan./abr., 2017. http:www.uel.br/revistas/informacao/ 
Roberta Rodrigues Faoro, Mírian Oliveira, Marcelo Faoro de Abreu O compartilhamento do conhecimento e a inovação nos relacionamentos interorganizacionais do tipo terceirização de tecnologia da informação

Ademais, em relação ao grau de novidade, foi percebida a inovação incremental em todas as empresas e somente na Totvs foi percebida inovação radical, sendo que as empresas Clientes da Totvs corroboram com esta inovação radical. Este achado pode ser explicado pelo estudo de Arnold, Fang e Palmatier (2011), o qual apresenta que a profundidade do conhecimento do cliente não se relaciona de forma significativa para o desempenho da inovação radical, mas se relaciona positivamente para o desempenho da inovação incremental. Já a diversidade de conhecimento do cliente se relaciona positivamente para o desempenho da inovação radical, enquanto a diversidade de conhecimento do cliente se relaciona negativamente com o desempenho da inovação incremental.

\section{CONSIDERAÇÕES FINAIS}

Esta pesquisa teve como objetivo analisar a existência de relação entre o compartilhamento do conhecimento e a inovação nas relações interorganizacionais do tipo terceirização de TI. Para atingir o objetivo do estudo foi realizado um estudo de múltiplos casos, assim sendo, foram no total quatro casos com doze empresas e 38 entrevistados, ou seja, cada caso possui três empresas, sendo uma a empresa fornecedora do ERP e duas empresas clientes de diferentes portes e atuantes em segmentos distintos para tentar evitar, com isso, a possibilidade de existência de tendências relacionadas ao porte ou ao segmento de atuação das organizações estudadas. Sendo assim, observou-se uma relação positiva entre o compartilhamento do conhecimento e a existência de inovação no ambiente de terceirização de $\mathrm{Tl}$. Outro resultado, foi em relação à decisão da terceirização do ERP, ou seja, pôde-se perceber que os motivos das "empresas clientes" terceirizarem seus ERPs, podem influenciar o compartilhamento do conhecimento e a inovação. Quer dizer, a empresa cliente que tem como motivo principal a redução de custo, na maioria das vezes, compartilha conhecimentos com a fornecedora somente quando necessário, resultando 
Roberta Rodrigues Faoro, Mírian Oliveira, Marcelo Faoro de Abreu O compartilhamento do conhecimento e a inovação nos relacionamentos interorganizacionais do tipo terceirização de tecnologia da informação

com isso, menos inovações através da terceirização do ERP. Contrapondo-se a isso, a empresa cliente que tem outros motivos, além da redução de custos, como exemplo, o foco no negócio ou acesso à expertise do fornecedor, tem maior compartilhamento do conhecimento, logo, maior inovação através da terceirização do ERP.

Sendo assim, pôde-se concluir, com esta pesquisa, que existem casos de inovações nas 12 empresas estudadas, que foram relacionadas com o compartilhamento do conhecimento nas relações interorganizacionais do tipo terceirização de TI. Dessa forma, pôde-se observar no estudo algumas empresas com maior intensidade e outras com menor intensidade na relação entre o compartilhamento do conhecimento e a inovação.

Além disso, este estudo buscou pesquisar os tipos de conhecimentos compartilhados, ou seja, conhecimento tácito e explícito e os mecanismos formais, informais e tecnológicos que auxiliam no compartilhamento do conhecimento. Neste sentido, pôde-se concluir que as empresas fornecedoras têm maior interesse em compartilhar conhecimentos com seus clientes, isto deve-se ao fato, que os conhecimentos compartilhados podem ser absorvidos e incorporados no seu produto final, ou seja, podem ocorrer melhorias no ERP baseado no compartilhamento do conhecimento. Ainda assim, pôde-se observar que as empresas trocam conhecimentos tanto tácito quanto explícito, sendo que as diferenças entre elas são: as empresas fornecedoras tendem a formalizar todo o conhecimento compartilhando através de um software, desta forma, priorizam o conhecimento explícito. Já, a maioria das empresas clientes prefere socializar os conhecimentos por meio de conversas informais.

Sendo assim, tanto do ponto de vista acadêmico quanto gerencial, esta pesquisa contribuiu por gerar evidências empíricas sobre a relação entre o compartilhamento de conhecimento e a inovação nos relacionamentos interorganizacionais do tipo terceirização de tecnologia da informação. Por outro lado, a pesquisa apresentou, como principal limitação, a quantidade de empresas por segmento, sendo assim, novos estudos podem ser feitos com o 
Roberta Rodrigues Faoro, Mírian Oliveira, Marcelo Faoro de Abreu O compartilhamento do conhecimento e a inovação nos relacionamentos interorganizacionais do tipo terceirização de tecnologia da informação

intuito de corroborar ou até mesmo expandir este estudo, pois se tratando de um estudo de múltiplos casos, os resultados não podem ser generalizados.

Por fim, a realização deste estudo possibilitou a visualização de oportunidades de pesquisas, tanto relacionadas diretamente com este trabalho como novos assuntos que emergiram da pesquisa exploratória. Abaixo estão relacionados alguns trabalhos a serem realizados no futuro com base nas motivações oriundas deste artigo:

- Realizar pesquisa quantitativa com o objetivo de validar e expandir os resultados aqui encontrados.

- Aplicar o instrumento de pesquisa, entre empresas fornecedoras de $\mathrm{TI}$ e empresas clientes do mesmo segmento, para analisar se os resultados do compartilhamento do conhecimento e da inovação podem ser diferentes por segmentos. Neste sentido, pesquisar empresas fornecedoras de outros segmentos.

- Aplicar o instrumento de pesquisa, entre organizações públicas e privadas para verificar se existem diferenças nos resultados aqui encontrados.

\section{REFERÊNCIAS}

ALAVI, M.; LEIDNER, D.E. Knowledge management and knowledge management systems: Conceptual foundations and research issues. MIS Quarterly, v. 25, n. 1, p. 107-136, 2001.

ARGOTE, L. Organizational learning: creating, retaining, and transferring knowledge. Norwell, Massachusetts: Kluwer Academics, 1999.

A.T. KEARNEY. Global Services Location Index. A Wealth of Choices: From Anywhere on Earth to No Location at All, 2014. Disponivel em: $<$ https://www.atkearney.com/documents/10192/5082922/A+Wealth+of+Choices .pdf/61c80111-41b2-4411-ad1e-db4a3d6d5f0d>. Acesso em: 15 dez. 2015.

BANDYOPADHYAY, S.; PATHAK. P. Knowledge sharing and cooperation in outsourcing projects - A game theoretic analysis. Decision Support Systems, v. 43, p. 349-358, 2007. 
Roberta Rodrigues Faoro, Mírian Oliveira, Marcelo Faoro de Abreu O compartilhamento do conhecimento e a inovação nos relacionamentos interorganizacionais do tipo terceirização de tecnologia da informação

BARDIN, L. Análise de Conteúdo. Lisboa: Edições 70, 2006.

BAREGHEH, A.; ROWLEY, J.; SAMBROOK, S. Towards a multidisciplinar definition of innovation. Management Decision, v. 47, n. 8, p. 1323-1339, 2009.

BOCK, G.-W.; LEE, J.-N.; ZMUD, R. W. Behavioral intention formation in knowledge sharing. MIS Quarterly, v. 29, n. 1, p. 87-111, 2005.

BROZEN, Y. Invention, Innovation and Imitation. Anterican Economic Review, v. 41, p. 239-57, 1951.

CRESWELL, J.W. Projeto de pesquisa: métodos qualitativo, quantitativo e misto. 2. ed. Porto Alegre: Artmed, 2007.

DAMANPOUR, F.; GOPALAKRISHNAN, S. Theories of organizational structure and innovation adoption: the role of environmental change. Journal of Engineering and Technology Management, v. 5, n. 1, p. 1-24, 1998.

DAVENPORT, T. H. Information Ecology. New York: Oxford University Press, 1997.

DAVIS, B.; RIGGS, B. Knowledge Management: Get Smart. Information Week, april, p. $40-46,1999$

DRUCKER, P. The Age of Discontinuity. Nova lorque: Harper and Row, 1969. 393 páginas.

DUBÉ, L.; PARÉ, G. Rigor in information systems positivist case research: current practices, trends, and recommendations, MIS Quarterly, v.27, n.4, p.597-635, 2003.

DUTTA, D. K. Inter-Organizational Relationships and Firm Performance: Impact of Complementary Knowledge and Relative Absorptive Capacity. Journal of Management Policy and Practice, v. 13, n. 2, p. 46-55, 2012.

DYER, J.H.; NOBEOKA, K. Creating and managing a high performance Knowledge-sharing network: the Toyota case. Strategic Management Journal, v. 21, n.3, p. 345-367, 2000.

GARCIA, R.; CALANTONE, R. A critical look at technological innovation typology and innovativeness terminology: A literature review. The Journal of Product Innovation Management, v. 19, n. 2, p. 110-132, 2002. 
Roberta Rodrigues Faoro, Mírian Oliveira, Marcelo Faoro de Abreu O compartilhamento do conhecimento e a inovação nos relacionamentos interorganizacionais do tipo terceirização de tecnologia da informação

GARTNER. Gartner Says Worldwide IT Outsourcing Market to Reach \$288 Billion in 2013. Press Release. STAMFORD, Conn., July 17, 2013. Disponível em: <http://www.gartner.com/newsroom/id/2550615>. Acessado em 24 de maio de 2017.

GOTTSCHALK, P. Research Propositions for Knowledge Management Systems Supporting It Outsourcing Relationships. The Journal of Computer Information Systems. v. 46, n. 3, p. 110-116, 2006.

GRANT, R.M. Toward a knowledge-based theory of the firm. Strategic Management Journal, v. 17, p. 109-122, 1996.

HAN, K.; MITHAS, S. The Real Savings From IT Outsourcing. MIT Sloan Management Review, Winter, v. 55, n. 2, p. 16, 2014.

HARDY, C.; PHILLIPS, N.; LAWRENCE, T. B. Resources, knowledge and influence: The organizational effects of interorganizational collaboration. Journal of Management Studies, v. 40, n. 2, p. 321, 2003.

HSU, I.-C. Enhancing employee tendencies to share knowledge-case studies of nine companies in Taiwan. International Journal of Information Management, v. 26, n. 4, p. 326-338, 2006.

HWANG, Y.; KIM, D. J. Understanding affective commitment, collectivist culture, and social influence in relation to knowledge sharing in technology mediated learning. IEEE Transactions on Professional Communication, v. 50, n. 3, p. 232-248, 2007.

JANOWICZ-PANJAITAN, M.; NOORDERHAVEN, N. G. Formal and informal interorganizational learning within strategic alliances. Research policy, v. 37. n. 8, p. 1337-1355, 2008.

KING, W. R. Integrating Knowledge Management into IS Strategy. Information Systems Management, v. 16, n. 4, p. 70-72, 1999.

LACITY, M. C. et al. A review of the IT outsourcing empirical literature and future research directions. Journal of Information Technology, v. 25, n.4, p. 395-433, 2010.

LAWSON et al. Knowledge Sharing in Interorganizational Product Development Teams: The Effect of Formal and Informal Socialization Mechanisms. Product Development \& Management Association, v. 26, n. 2, p. 156-172, 2009.

LEE, J.-N. The impact of knowledge sharing, organizational capability and partnership quality on IS outsourcing success. Information \& Management, v. 38, n. 5, p. 323-335, 2001. 
Roberta Rodrigues Faoro, Mírian Oliveira, Marcelo Faoro de Abreu O compartilhamento do conhecimento e a inovação nos relacionamentos interorganizacionais do tipo terceirização de tecnologia da informação

LEE, J.-N.; MIRANDA, S. M.; KIM, Y.-M. IT Outsourcing Strategies: Universalistic, Contingency, and Configurational Explanations of Success. Information Systems Research, v. 15, n. 2, p. 110-131, 2004.

LEE, K. C.; LEE, S.; KANG, I.W. KMPI: measuring knowledge management performance. Information and Management, v. 42, n. 3, p. 469-482, 2005.

LYNSKEY, M.J. The transfer of resources and competencies for developing technological capabilities-the case of Fujitsu-ICL. Technology Analysis \& Strategic Management, v. 11, n.3, p. 317-336, 1999.

MANI, D.; BARUA, A.; WHINSTON, A. An empirical analysis of the impact of information capabilities design on business process outsourcing performance. MIS Quarterly, v. 34, n. 1, p. 39-62, 2010.

MCDONOUGH, E.F.; KAHN, K.B.; BARCZAK, G. An Investigation of the Use of Global, Virtual, and Colocated New Product Development Teams. Journal of Product Innovation Management, v. 18, n. 2, p. 110-120, 2001.

MERRIAM, S. B. Qualitative Research and Case Study Applications in Education: Revised and Expanded from Case Study Research in Education. São Francisco: Jossey-Bass, 1998.

MONCZKA, R.M.; TRENT, R.J.; HANDFIELD, R. B. Purchasing and Supply Chain Management. Cincinnati: South-Western, 2000.

NELSON, R.; WINTER, S. An Evolutionary Theory of Economic Change. Cambridge, MA: Belknap, 1982.

NONAKA, I. A dynamic theory of organizational knowledge creation. Organization Science, v. 5, n. 1, p. 14-37, 1994.

NONAKA, I.; TAKEUCHI, H. The Knowledge-Creating Company: how Japanese Companies Create the Dynamics of Inovation. Nova York: Oxford University Press, 1995.

NONAKA, I.; VON KROGH, G.; VOELPEL. S. Organizational Knowledge Creation Theory: Evolutionary Paths and Future Advances. Organization Studies, v. 27, n. 8, pp. 1179-1208, 2006.

NORD, W.; TUCKER, S. Implementing Routine and Radical Innovations. Lexington, MA: Lexington Books, 1987. 
Roberta Rodrigues Faoro, Mírian Oliveira, Marcelo Faoro de Abreu O compartilhamento do conhecimento e a inovação nos relacionamentos interorganizacionais do tipo terceirização de tecnologia da informação

OECD. Manual de Oslo: diretrizes para coleta e interpretação de dados sobre inovação. 3. ed. Tradução de Finep. Rio de Janeiro: OCDE; Eurostat; Finep, 2005.

ORANGE, G. et al. Local government and social or innovation value. Transforming Government: People, Process \& Policy, v. 1, n. 3, p. 242-254, 2007.

OWEN-SMITH, J.; POWELL, W. Knowledge networks as channels and conduits: The effects of spillovers in the Boston biotechnology community. Organization Science, v. 15, n. 1, p. 5-21, 2004.

PARDO et al. Knowledge sharing in cross-boundary information system development in the public sector. Inf Technol Manage, v. 7, s/n, p. 293-313, 2006.

PLESSIS, M. D. The role of knowledge management in innovation. Journal of Knowledge Management, v. 11 n. 4, p. 20-29, 2007.

POLANYI, M. Tacit Dimension. Chicago, IL: University of Chicago Press, 1983.

POPADIUK, S.; CHOO, C. W. Innovation and knowledge creation: How are these concepts related? International Journal of Information Management, v. 26, p. $302-312,2006$.

POPPO L.; ZHOU K.Z.; ZENGER, T. Examining the conditional limis of relational governance: Specialized assets, performance ambiguity, and longstanding ties. Journal of Management Studies, v. 45, n. 7, p. 1195-1216, 2008.

POWELL, W. W.; BRANTLEY, P. Competitive cooperation in biotechnology: learning through networks? In: NOHRIA, N.; ECLLES, R. G. Network and organizations. Harvard: Harvard Business School Press, p. 366-394, 1992.

QI, C.; CHAU, P. Y. K. Relationship, contract and IT outsourcing success: Evidence from two descriptive case studies. Decision Support Systems, v. 53, s/n, p. 859-869, 2012.

SATO, Karoline Aparecida Scroch; SILVA, Helena de Fátima Nunes; DRAGO, Isabela; BREDA, Sônia Maria. Criação e compartilhamento de conhecimento: perfis profissionais para o futuro da indústria paranaense. Informação \& Informação, Londrina, v. 20, n. 3, p. 297-326, set./dez. 2015.

SCHUMPETER, J.A. The Theory of Economic Development. Cambridge, MA: Harvard University Press, 1934. 
Roberta Rodrigues Faoro, Mírian Oliveira, Marcelo Faoro de Abreu O compartilhamento do conhecimento e a inovação nos relacionamentos interorganizacionais do tipo terceirização de tecnologia da informação Row, 1950.

Capitalism, Socialism and Democracy. New York, NY: Harper \& . Teoria do desenvolvimento econômico: uma investigação sobre lucros, capital, crédito, juro e o ciclo econômico. São Paulo: Nova Cultural, 1982.

SCOTT, J. E.; KAINDL, L. Enhancing functionality in an enterprise software package. Information and Management, v. 37, s/n., p. 111-122, 2000.

SENGUPTA, K.; ZVIRAN, M. Measuring user satisfaction an outsourcing environment. IEEE transactions on Engineering Management, v. 44, n. 4, p. 414-421, 1997.

SHER, P.; LEE, V. Information technology as a facilitator for enhancing dynamic capabilities through knowledge management. Information Management, v. 41, n.8, p. 933-945, 2004.

SOPER, D. S.; DEMIRKAN, H.; GOUL, M. An interorganizational knowledgesharing security model with breach propagation detection. Information Systems Frontiers, v. 9, n. 5. p. 469-479, 2007.

TEECE, D.J. Strategies for managing knowledge assets: the role of firm structure and industrial context. In: Nonaka, I., Teece, D. (Eds.), Managing Industrial Knowledge: Creation, Transfer and Utilization. London, UK: Sage Publications, p. 125-144, 2001.

THOMPSON'S, V.A. Bureaucracy and innovation. Administrative Science Quarterly, v. 10, p. 1-20, 1965.

TRIVINÕS, A.N.S. Introdução à pesquisa em Ciências Sociais: a pesquisa qualitativa em educação. São Paulo: Atlas, 1987.

VAN MAANEN, J. Reclaiming qualitative methods for organizational research: A preface. Administrative Science Quarterly, v. 24, n.4, p. 520-526, 1979.

VARIS, M.; LITTUNEN, H. Types of innovation, sources of information and performance in entrepreneurial SMEs. European Journal of Innovation Management, v. 13, n. 2, p. 128-154, 2010.

VERGARA, S. C. Métodos de pesquisa em administração. São Paulo: Atlas, 2005.

VIVEK, G.; SUSHIL. IS Outsourcing Relationship: A Review of Literature. Advances In Management, v. 7, n. 9, p. 33-43, 2014. 
Roberta Rodrigues Faoro, Mírian Oliveira, Marcelo Faoro de Abreu O compartilhamento do conhecimento e a inovação nos relacionamentos interorganizacionais do tipo terceirização de tecnologia da informação

WANG, C.; HAN, Y. Linking properties of knowledge with innovation performance: the moderate role of absorptive capacity. Journal of Knowledge Management, v. 15, n. 5, p. 802-819, 2011.

WILLEM, A.; BUELENS, M. Knowledge sharing in inter-unit cooperative episodes: The impact of organizational structure dimensions. International Journal of Information Management, v. 29, n. 2, p. 151-160, 2009.

WILLIAMS, R.T. Narratives of knowledge and intelligence: beyond the tacit and explicit. Journal of Knowledge Management, v. 10, n. 4, p. 80-99, 2006.

YIN, R. K. Estudo de caso: planejamento e métodos. 2. ed. Porto Alegre: Bookman, 2001.

YOON, Y.; IM, K.S. An Evaluation System for IT Outsourcing Customer Satisfaction Using the Analytic Hierarchy Process. Journal of Global Information Management, v. 13, n. 4, p. 55-78, 2005.

ZANINELLI, T. Gestão da inovação: considerações em torno do processo de desenvolvimento de serviços. Informação \& Informação, Londrina, v. 17, n.2, p. 133-155, maio/ago. 2012.

ZIVIANI, F.; FERREIRA, M. A. T.; NEVES, J. T. R. Fontes de informação para inovação no setor elétrico brasileiro. Informação \& Informação, Londrina, v. 20, n. 1, p. 162-182, jan./abr. 2015.

Title

Knowledge sharing and innovation in relationships interorganizational type of information technology outsourcing

\section{Abstract:}

Introduction: this paper presents an analysis of the sharing of knowledge and innovation in the inter-relationships of the type outsourcing of Information Technology.

Objective: analyze the existence of relationship between knowledge sharing and innovation in the inter-relationships of the type of outsourcing information technology (IT). Methodology: research is exploratory, qualitative and using as a strategy the multiple case study, which analyzed 12 companies in IT outsourcing environments, 4 suppliers of IT and IT 8 client companies. Data were collected through semi structured interviews, documentary and direct observation. Results: it can be noted that the sharing of knowledge showed contribute to the existence of innovation in IT outsourcing environment. The suppliers prioritize the codification of shared knowledge, while customers prefer the sharing of knowledge through informal conversations. It was identified that the motives for outsourcing IT may be related to the sharing of 
Roberta Rodrigues Faoro, Mírian Oliveira, Marcelo Faoro de Abreu

O compartilhamento do conhecimento e a inovação nos relacionamentos interorganizacionais do tipo terceirização de tecnologia da informação

knowledge and innovation. Conclusions: this research contributes to the advancement of knowledge about the knowledge and innovation sharing phenomena in outsourcing of IT environments.

Keywords: Interorganizational Knowledge Sharing. Innovation. Information technology.

\section{Titulo}

Intercambio de conocimientos e innovación en las relaciones interorganizational tipo de outsourcing tecnología de la información

\section{Resumen:}

Introducción: en este trabajo se presenta un análisis de la puesta en común de conocimientos y la innovación en las interrelaciones de la externalización tipo de tecnología de la información. Objetivo: analizar la existencia de relación entre el intercambio de conocimientos y la innovación en las interrelaciones del tipo de tecnología de la información externalización (TI). Metodología: la investigación es exploratoria, cualitativa y utilizando como estrategia el estudio de casos múltiples, donde se analizaron 12 empresas de $\mathrm{TI}$ en entornos de externalización, 4 proveedores de $\mathrm{TI}$ y 8 empresas clientes de TI. Los datos fueron recolectados a través de entrevistas semiestructuradas, documentos y observación directa. Resultados: cabe señalar que el intercambio de conocimientos mostró contribuyen a la existencia de la innovación en el entorno de TI de externalización. Los proveedores dan prioridad a la codificación de conocimiento compartido, mientras que los clientes prefieren el intercambio de conocimientos a través de conversaciones informales. Se encontró que las razones para la externalización de TI pueden estar relacionados con el intercambio de conocimientos y la innovación. Conclusiones: esta investigación contribuye al avance del conocimiento sobre el conocimiento y la innovación de intercambio de fenómenos en la externalización de los entornos de TI.

Palabras clave: El intercambio de conocimientos interorganizational. la innovación. tecnología de la información.

Recebido: 12.02 .2016

Aceito: 25.03.2017 